\title{
Experimental observations on sorting patterns of heterogeneous sediment mixtures in low constrained flows
}

\author{
Costanza Carbonari ${ }^{1,2,}$, ,Francesco Tanganelli ${ }^{1,2}$, Alain Recking ${ }^{1}$ and Luca Solari ${ }^{2}$ \\ ${ }^{1}$ Irstea, Centre de Grenoble, UR ETGR, 2 Rue de la Papeterie, 38402 St Martin d'Hères, France \\ ${ }^{2}$ Dipartimento di Ingegneria Civile e Ambientale, Università degli Studi di Firenze, Via di S. Marta 3, \\ 50139 Firenze, Italy
}

\begin{abstract}
Field and laboratory investigations indicate that gravel bed rivers with bimodal grain size distribution and low lateral confinement, such as in the case of braided or multi-channel rivers, can present simultaneously active channel variations, both in the planimetric and altimetric directions, together with planimetric and vertical sorting. Such aspects were reproduced in new flume experiments considering three flow confinements with characteristic aspect ratios from about 80 to 5 . Three long runs of about 60 hours were carried out under constant feeding rate conditions, with a bimodal mixture of natural sediments, a fixed flume slope of $3.18 \%$, and width imposed by lateral walls from $0.50 \mathrm{~m}$ to 0.12 $\mathrm{m}$. We present here the results obtained with the first run, with a width of $0.5 \mathrm{~m}$. We observed fluctuations of bed surface granulometric composition, bed slope, and outlet solid discharge; each of which with its own periodicity. In particular, the rearrangement of the bed surface texture was rapid. Cyclic bed states were observed: a stage of coarsening and aggradation corresponding to low values of outlet solid discharge; a stage of fining and degradation concomitant to high values of transport rate. The other two on-going runs (narrower configurations) aim at investigate the effect of lateral confinement on the morphodynamics of the system.
\end{abstract}

\section{Introduction}

River beds present different size fractions of sediments which differently interact with flow: during bed load transport, heterogeneous sediment mixtures undergo the process of sorting so that the grain size distribution of river beds displays significant spatial structures, both in the surface texture and in the stratigraphy, giving rise to several kinds of patterns occurring at different spatial scales (river profile, reach, unit). These patterns typically develop both in the longitudinal direction (e.g. downstream fining) and in the vertical direction (e.g. armoring). Sorting patterns are detected in all stream morphologies, from steeper mountain streams with high later confinement to lowland rivers. Moreover, they play a decisive role when considering river bed forms dominated by sorting [1], as with bed load sheets [2,3].

\footnotetext{
* Corresponding author: costanza.carbonari@irstea.fr
} 
The case of gravel bed rivers typically characterized by bimodal sediment mixtures is of particular interest since sorting can concern the vertical grain size structure as well as those in the streamwise and spanwise directions. In fact, in this kind of streams the presence of an alluvial bed with mobile banks, moderate slope, and a weak mobility of the coarse sediment fraction makes it possible for the active bed to evolve also in the plane, whereas it is not the case for more confined, steeper streams. In weakly confined gravel bed rivers is therefore possible to observe both slope and width adjustments and their complex mutual dependence on vertical and planimetric sediment sorting [4]. Active channel variations and heterogeneous sediment sorting also interact with bed forms, affecting their formation, morphology and migration, as has been observed in bars [5, 6] and in braided patterns [7].

Narrow flume experiments have demonstrated interactions between channel slope adjustments, vertical sorting, and fluctuations of bed load transport rate [3, 8]. In particular, Recking et al. [3] and Bacchi et al. [8] observed significant fluctuations of slope and outlet solid discharge, despite the constant feeding rate conditions. Furthermore they observed that during stages characterized by increasing bed slope, a paved state of the bed took place and concurrently the outlet solid discharge decreased; on the other hand, when peaks of outlet solid discharge associated with the passage of bed load sheets [3] the bed slope decreased. Conversely, only few investigations [7] studied configurations with low lateral constraint with regard to grain sorting, observing absence of stationarity of bed forms and cyclicity of bed states accompanied by planimetric variations of the channel.

The present study aims at identify grain sorting processes in the case of different degrees of flow confinement, considering first low constraint flows (with a width to average flow depth ratio of about 80 in the configuration with the lowest confinement, of about 20 for the intermediate configuration) and then a confined flow (with a width to average flow depth ratio of about 5). Results of the first experiment are presented here.

\section{Materials and methods}

\subsection{Experimental settings}

The experiments were conducted in a 6-m-long flume with a maximum width of $0.5 \mathrm{~m}$. The flume bed consisted of a layer of the sediment mixture used for the inlet solid discharge; with bimodal grain size distribution: the coarse fraction is characterized by a $\mathrm{D}_{50}$ equal to $1.8 \mathrm{~mm}$ and it constitutes the $60 \%$ of the mixture, the fine fraction has a $\mathrm{D}_{50}$ equal to 0.7 $\mathrm{mm}$ and it constitutes the $40 \%$ of the mixture. We performed constant feeding rate conditions both for water and solid discharges with the values of $\mathrm{Q}=0.6 \mathrm{l} / \mathrm{s}$ e $\mathrm{Q}_{\mathrm{s}}=8 \mathrm{~g} / \mathrm{s}$.

At the upstream section, the sediments enter the flume by means of a hopper; the solid discharge is controlled through the opening of the hopper and through the speed of the conveyor belt, which transfers the sediments at the flume entrance. Sediments are collected at the flume outlet where the solid discharge is measured by weighing the exiting sediments in a given time interval. The slope of the flume is fixed at a value of $3.18 \%$. The outlet controlled section had a central rectangular opening.

During the experimental activity, three long runs were produced differing from one another in respect of the lateral constraint as indicated in the following table. 
Table 1. Main characteristics of the tree runs carried out.

\begin{tabular}{lccccr}
\hline \# Run & Flume width (cm) & Flume slope (\%) & Q in (l/s) & Qs in (g/s) & Time (h) \\
\hline 1 & 50 & 3.18 & 0.6 & 8 & 60 \\
2 & 25 & 3.18 & 0.6 & 8 & 60 \\
3 & 12 & 3.18 & 0.6 & 8 & 60 \\
\hline
\end{tabular}

\subsection{Measurements}

For the whole duration of each run, we monitored the following physical quantities, each of which with its own time interval for measurement:

- granulometric composition of the bed surface $(\Delta t=1$ minute);

- $\quad$ average bed slope $(\Delta t=30$ minutes);

- $\quad$ outlet solid discharge $(\Delta \mathrm{t}=10$ minutes);

- $\quad$ active channel width at three cross-sections ( $\Delta \mathrm{t}=10$ minutes).

During each experiment, we carried out topographic measurements in order to reconstruct the Digital Elevation Model of the bed, an example of which is shown in figure 1.

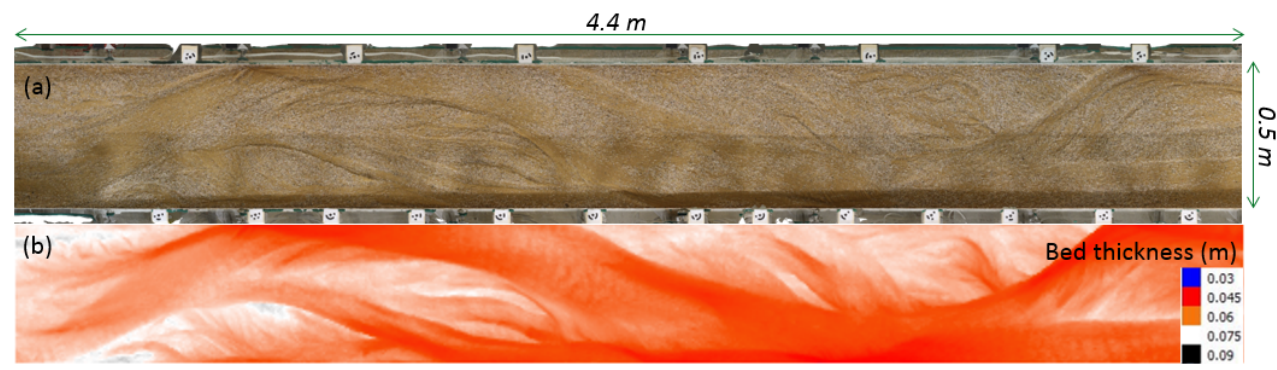

Fig. 1. (a) Orthorectified image of the analyzed area of the flume. (b) Digital Elevation Model obtained through Structure from Motion techniques, in particular the software Agisoft PhotoScan

In addition, we measured the surface texture of the bed and the 2-dimensional water surface velocity field. These measurements are carried out through a non-intrusive system based on photos taken by three cameras placed above the flume and fixed on a mobile system; the latter can move along a rail parallel to the flume. Furthermore, we performed manual measurements in several cross-sections monitoring the average bed elevation by means of a digital elevation gauge and the active channel width.

Throughout the entire duration of each run, we used one camera to take photos from which we determined the bed surface granulometric composition by means of image processing techniques. These photos were taken without interrupting the experiment, at a frequency of once per minute. We used the other two cameras for the sequence of photos by which we reconstructed the Digital Elevation Model of the bed using Structure from Motion techniques.

Bed surface texture analysis was possible because the two granulometric fractions have different colours, making it possible to separate coarse grains from fines since they differ one from another in their spectrum (figures 2 and 3). We are actually interested not by a strict separation of the two fractions, but rather by an identification of the patches of fines, which are characteristic of active transport. 


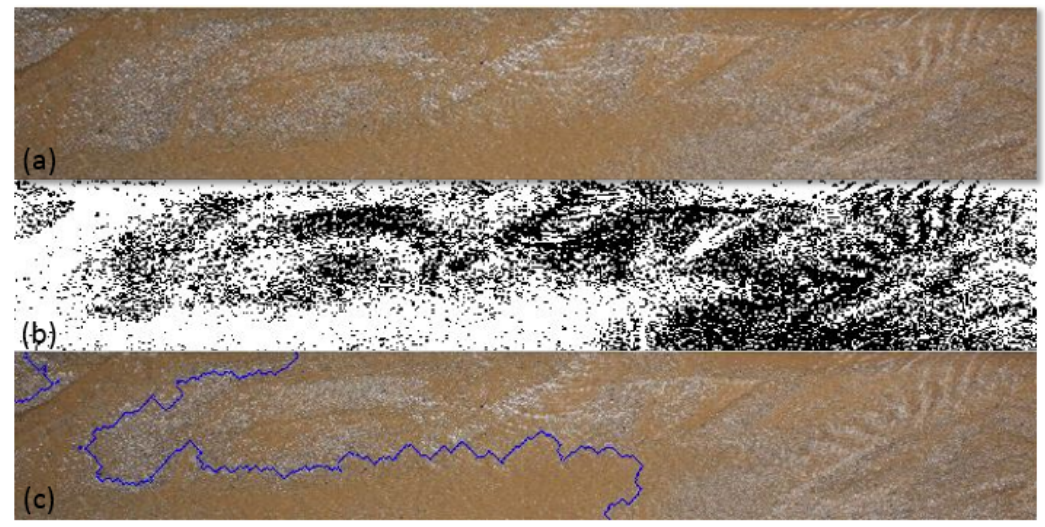

Fig. 2. The central part of the flume for the photos used to study the granulometric composition of the bed surface. (a) The original RGB image; (b) the binary image obtained from the blue channel extracted from the RGB image; in the binary image the two granulometric fractions (in white the fines, in black the coarses) are separated, is therefore possible to determine the composition of the bed surface and to perform morphological operations which identify sediments patterns. In panel (c) a patch of fines is delimited.

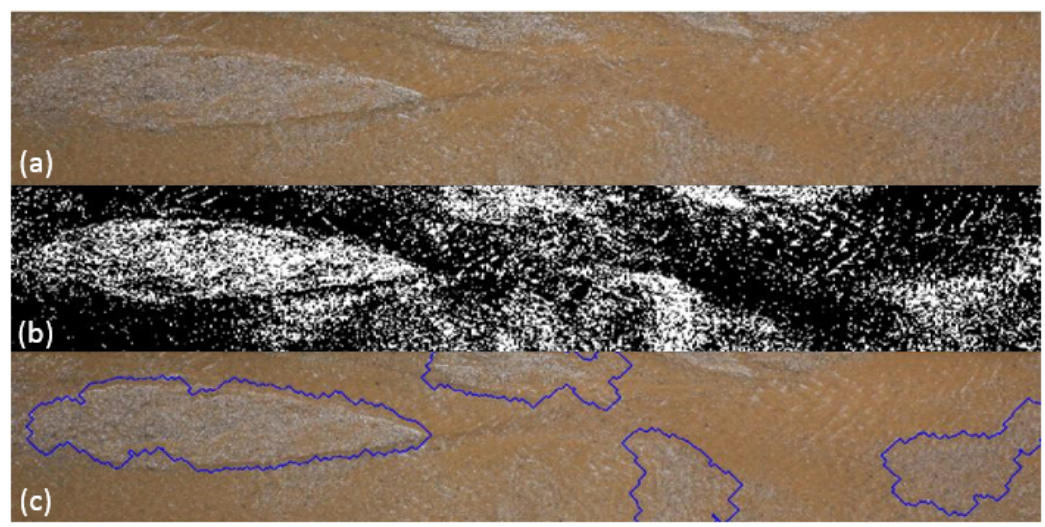

Fig. 3. (a) The original RGB image; (b) the binary image (in white the coarses, in black the fines) obtained from the blue channel extracted from the RGB image; (c) the patterns of coarse grains identified thanks to the morphological operations performed on the binary image.

Finally, always using the same cameras, we took pictures for short time intervals at a rate of 50 photos per second, framing a limited area of the flume. Thereby we were able to determine the 2-dimensional water surface velocity field. For this purpose, we dyed water and we employed precise seeding techniques; the method we used to obtain velocity field is the Large Scale Particle Image Velocimetry, in particular we used the software FudaaLSPIV $[9,10]$.

We conducted an experiment that lasted 60 hours and that was characterized by a configuration of the flume with a slope equal to $3.18 \%$ and a maximum width of $0.5 \mathrm{~m}$ (table 1). This configuration is the one with the lowest lateral confinement; in the present work, we reported the outcomes concerning the aforementioned experiment, while the other two experiments presented in table 1 are on-going. 


\section{Preliminary results}

Experimental observations have revealed rapid dynamics of channels' self-formation accompanied by sorting processes. Bed states were characterized by the presence of multiple bars and braided patterns, whose formation and destruction generally took place rapidly producing on the bed surface well-defined patches of coarse sediments and fines. We give an idea of such a rapid evolution and rearrangement of the bed surface in figure 4 . It appears that the granulometric sorting of the bed surface is very efficient, whereas vertical sorting is almost completely absent: samples of the substrate at the end of the run have indeed the same composition of the initial bed state and of sediment fed.

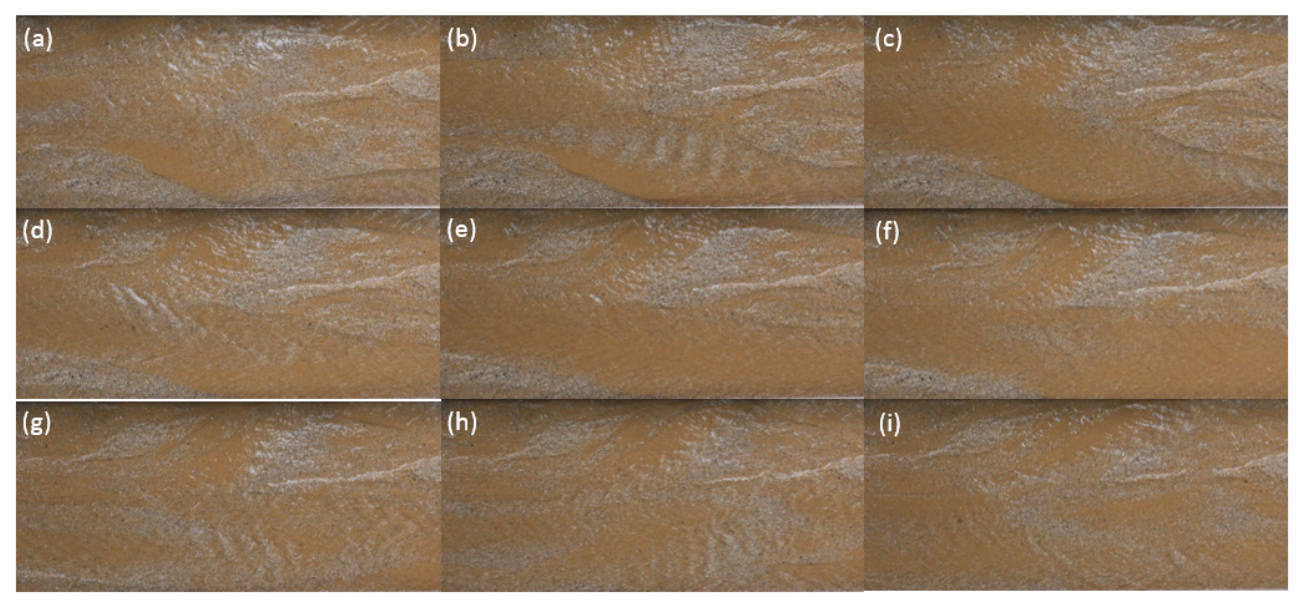

Fig. 4. Panels from (a) to (i) all frame the same area of the flume, in particular a zoom of the central part of the flume photographed to study the granulometric composition of the bed surface. These photos are taken every minute and they revealed how rapid is the rearrangement of the bed surface in terms paving/fining processes.

Results concern the evolution over time of the monitored physical quantities provided for in section 2.2.

Albeit the run was conducted under constant feeding rate conditions, we observed significant fluctuations of the percentage of the granulometric fractions composing the surface texture (figure 5a), the bed slope, and the outlet solid discharge (figure 5b). These fluctuations of the percentage of the coarse fraction of the bed surface, the slope, and the solid discharge were characterized by periodicities confirmed by Fourier analysis in the frequency domain. The evolution over time of the percentage of the coarse fraction was in opposition with the time trend of the sum of the surfaces covered by patches of fines, the sum of the aforementioned areas was expressed in terms of percentage of the total bed surface as well (figure 5a); patches of fine sediments constituted the zones where the channel was more active (in terms of transport rate).

It therefore turns out that when the patches of fines covered a wide area of the bed, the bed slope was low, corresponding to stages of fining and degradation of the bed; we observed indeed a concomitant increase of the outlet solid discharge. On the contrary, when the patches of fines covered a small area of the bed, a coarsening and aggradation bed state took place, in conjunction with a decrease of the outlet solid discharge; during this stage, the bed was characterized by higher values of the slope. 


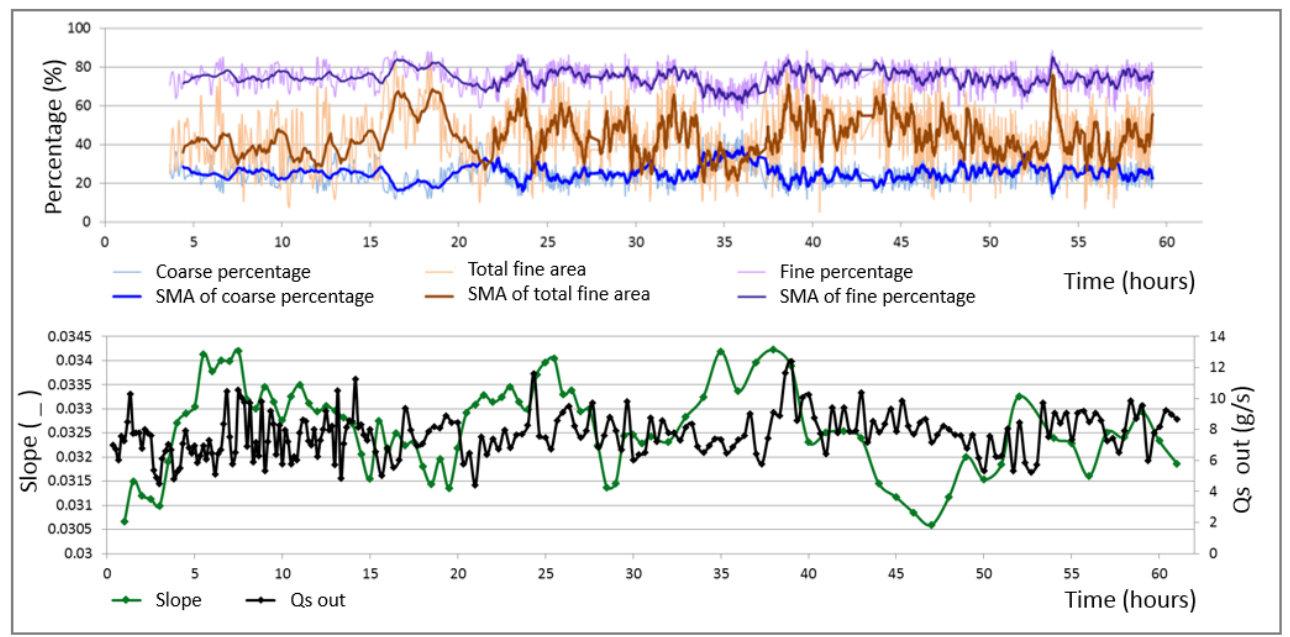

Fig. 5. (a) Time series of the percentage of the coarse fraction of the bed surface, the fine fraction of the bed surface, and the total surface covered by patches of fines; Simple Moving Average (SMA) of each quantity is shown as well. (b) Time series of the average bed slope and the outlet solid discharge.

These preliminary results confirm that, similarly to what was demonstrated in constrained flows, large bedload fluctuations also occur in non-constrained flows. However, they are not due to (only) vertical sorting and slope fluctuation, but also large bed surface sorting producing highly mobile patches of fines. The coming results with same input conditions will show how this surface process is affected when the width is reduced. Hopefully this protocol will help us to better understand the vertical versus horizontal sorting effects in the process of transport.

The support from the National Research Institute of Science and Technology for Environment and Agriculture, Center of Grenoble for carrying out the research work is thankfully acknowledged. We thank the two anonymous reviewers whose comments and suggestions are useful for further analysis.

\section{References}

1. G. Seminara, Effect of grain sorting on the formation of bedform, Applied Mechanics Reviews, 48 (1995)

2. J.G.Venditti, J.M. Nelson, W.E. Dietrich, The domain of bedload sheets, Proceedings of Marine and River Dune Dynamics III, International Workshop, University of Leeds, UK, 315-321 (2008)

3. A. Recking, P. Frey, A. Paquier, P. Belleudy, An experimental investigation of mechanisms involved in bed load sheet production and migration, Journal of Geophysical Research (2009)

4. T.E. Lisle, J.M. Nelson, J. Pitlick, M.A. Madej, B.L. Barkett, Variability of bed mobility in natural, gravel-bed channels and adjustments to sediment load at local and reach scales, Water Resources Research, 36, 3743-3755 (2000)

5. T.E. Lisle, H. Ikeda, F. Iseya, Formation of stationary alternate bars ina steep channel with mixed-size sediment: a flume experiment, Earth Surface Processes and Landforms, 16, 463-469 (1991)

6. S. Lanzoni, Experiments on bar formation in a straight flume 2. Graded sediment, Water Resources Research, 36, 3351-3363 (2000) 
7. P. Leduc, Etude experimentale de la dynamique sedimentaire des rivieres en tresses, Phd Thesis (2013)

8. V. Bacchi, A. Recking, N. Eckert, P. Frey, G. Piton, M. Naaim, The effects of kinetic sorting on sediment mobility on steep slopes, Earth Surface Processes and Landforms (2014)

9. J. Le Coz, M. Jodeau, A. Hauet, B. Marchand, R. Le Boursicaud, Image-based velocity and discharge measurements in field and laboratory river engineering studies using the free Fudaa-LSPIV software, Proceedings of the International Conference on Fluvial Hydraulics, RIVER FLOW, 1961-1967 (2014)

10. M. Muste, A. Hauet, C. Legout, H.C. Ho, Capabilities of large-scale particle image velocimetry to characterize shallow free-surface flows, Advances in Water Resources 70 160-171 (2014) 